\title{
Design and Development of Unity3D Game Engine-Based Smart SNG (Social Network Game)
}

\author{
Jae-Hwan Bae ${ }^{1}$ and Ae-Hyun Kim ${ }^{2}$ \\ 1, First Author Department of Game Engineering, TongMyong University, South Korea \\ *2,Corresponding Author Department of Industrial Design, \\ Dong-A University, South Korea \\ ${ }^{1}$ bjhmail@tu.ac.kr, ${ }^{2}$ kah08@dau.ac.kr
}

\begin{abstract}
As the number of smartphone users has been largely increased, games are highlighted because of their roles to promote the correlations by strengthening functions of SNS (Social Network Service). The SNG(Social Network Service) connects many people who don't know each other's around the world under one theme, therefore, becomes a communication tool for establishing a new human network. The games provided by social network service have increasingly drawn attention. In particular, a continuous expanding is exhibited in a sale along with the increase of users of SAS service, suggesting that the game industry is further activated. The grow h of social network game market shows a multi-platform expansion of social network game bydinking the offlonline of mobile app store and web one. However, the problem of it is a lack of related experiences and developers. And the current social network gamery are mostly created in a flash-based form, thus, there are problems in terms of multi-platform support and limits of $3 D$ games. Therefore, the Unity3D Engine developed by Unity Technologies, an engine which capable of making high-quality games in relatively easy and fast way used to overcome limits of $2 D$ social newrork games, and design and develop the $3 D$ social network game( $(S N G)$ using $3 D$ multi-platform engine unity. This is expected to contribute to an activation of the multi-platform-based smart SNG game market.
\end{abstract}

Keywords: $\mathrm{SNG}$, Unity3 3, Game Engine, Game Design, Smart Game

\section{Introduction}

\subsection{SNG status}

As The total number of domestic Smartphone subscribers passed 32 million in 2012, and the totalyumber of domestic subscribers to mobile communication, including Smartphone, recorded 52 million. Considering that the mobile communication service started from the late 80s, the Smartphone market has significantly grown and become very popular in a short period. Smartphone is not just a simple phone that only allows text messages and calls. With it, we can watch movies, read books, and communicate with people more smoothly. Moreover, we can enjoy games moving around freely that once we had to play in certain spaces such as in front of PCs or game machines. For mobile phones, many applications (App) are being developed as people who find simple fun in daily life increase. At this moment that various Apps are being developed, SNG has established itself as the best contributor creating high returns. Most of the games launched via KAKAO TALK in Korea and in collaboration with LINE in foreign countries such as Japan are occupying high ranks, and ANIPANG (developed by SUNDAYTOZ), the one that made a start of KAKAO TALK games, is still enjoying longevity in the App market where average life of App games is less than one year. In other words, SNG is the strong game App field that occupies high ranks not only in the Google Play Store but all the best sales categories. Actually, wherever there are people, we can easily find persons playing 
games with their Smartphones. Although those games come in various types, we cannot deny that these are SNG games linked with KAKAO TALK or LINE. Even only with this part, we can judge that the SNG market is growing rapidly and has high diversity.

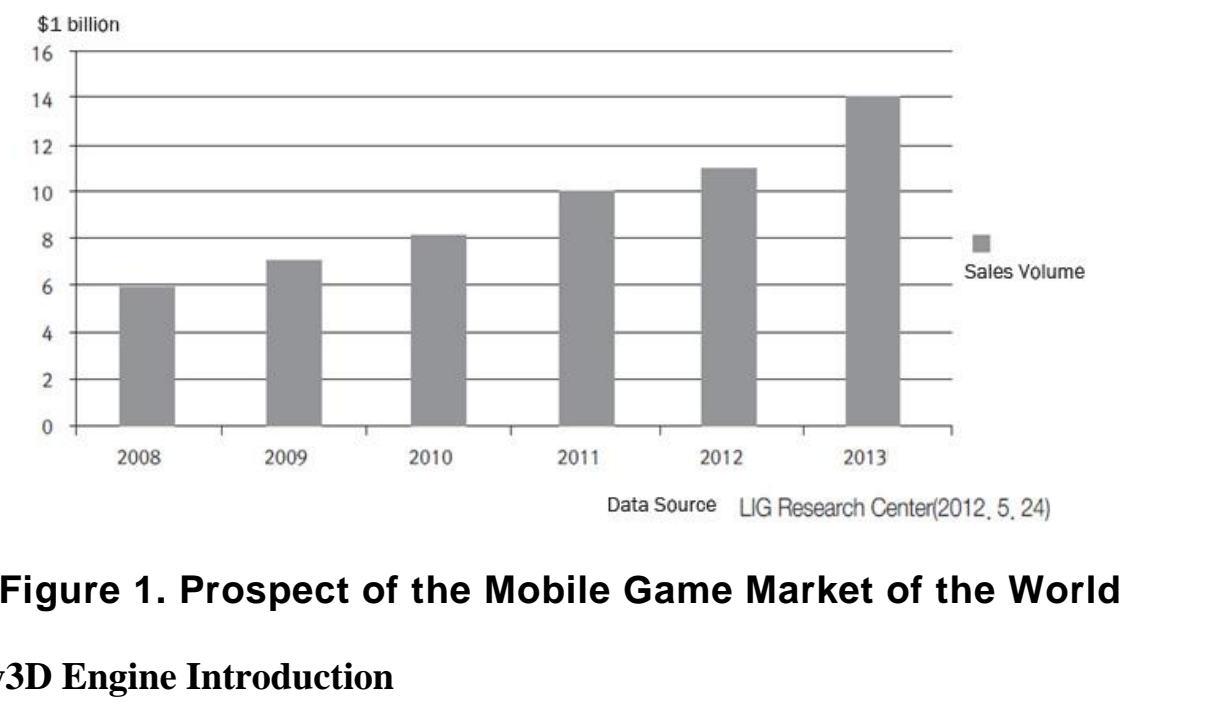

\subsection{Unity3D Engine Introduction}

It is a 3D game engine developed by Unity Technofogies, and is a data tool used to make not only games but contents $r$ real-time $3 \mathrm{D}$ animations, etc. It can be run on Windows and Macintosh OS X, and be used for detelopment on various platforms including Windows, Mac, Will, and iPhone, It can be used to make 3D web games utilizing a Unity web player plug-in. It is welr known in Korea, and is known as a tool for iPhone game production in the U.S. And because it provides easy and stable development environments compared to other game engines, it has a merit of a high development success tate. The most distinct characteristics of the Unity3D engine are easy development environments and multi-platform environments that can be easily conversed. Unity3D supports multi-platform environments such as Windows and Mac, Xbox360, PS3, Wii, Android, IOS, Explorer, Chrome, Safari, and Firefox, and can be run on Windolus and Mac [1-2].

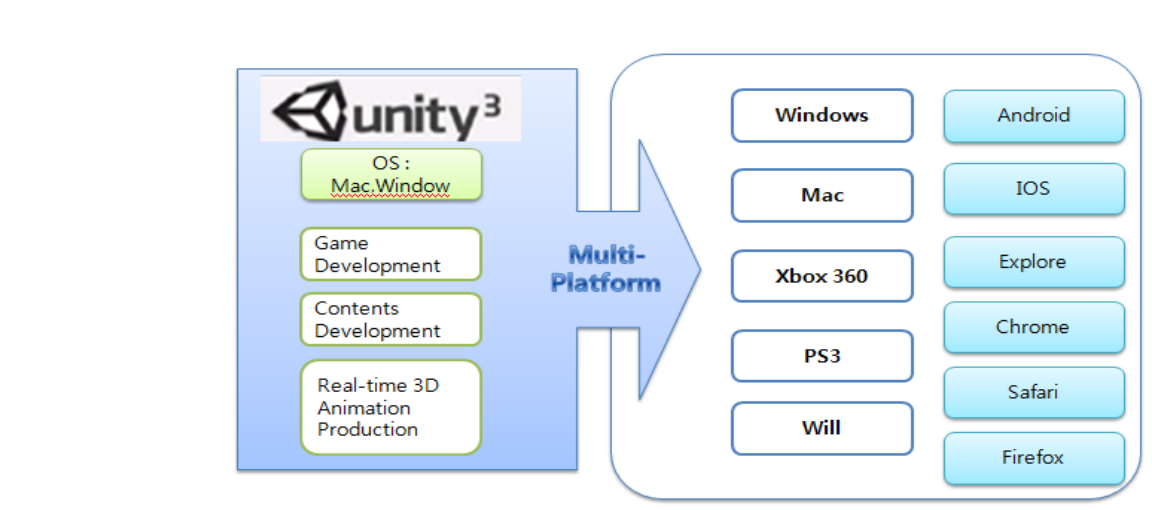

Figure 2. Unity3D Engine Multi-platform

Unity3D supports three script languages, Java Script, C\#, and Boo. They all enable quick interactions, can utilize the .NET library, and support database, regular expression, XML, networking, etc. Although it is generally recognized that scripting has limitations and is slow, because Unity3D shows very quick iteration time and operates being coupled with compiles of easy native codes, it is as fast as C++. And embodiment of Unity3D's Java Script operates at the same speed as C\# or Boo. Game logic is executed on the open 
source .NET platform, Mono. This provides the world level programing environment equipped with the best performance, speed, and flexibility. Only one line of code is enough to move and rotate objects and perform scaling. This is also applied to copying, removing, and modifying properties. Everything can be referred to by names, classes, tags, similarity, touches, etc. The functions of Unity3D have been integrated with all MonoDevelop.

\subsection{Multi-platform SNG Technology}

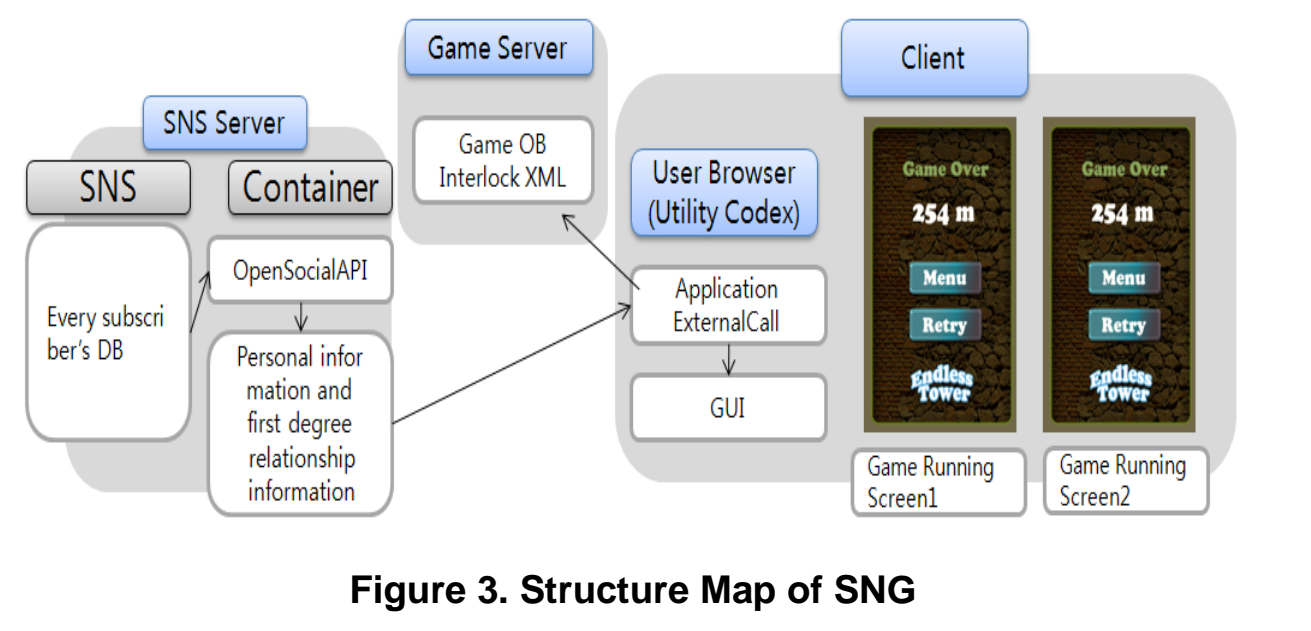

For scripting, Unity3D supports three languages, Java Script, C\#, and Boo, and these all can be used for one project. Thus, we cammake scripts freely, being undeterred by a certain language. However only one language can be used within one script. One thing we must know first when making scripts using Unity3D is References that enable Overriding. Among them, the most important things are Update, LateUpdate, FixedUpdate, Awake, and Start. The Update function is called for every frame when the script is on. We gan use Time.deltaTime, the most frequently used function in the script, when we want to know the past time. The LateUpdate function is also called for every frame, and is called after every Update function is called. The FixedUpdate function is also called for every frame, and is used in substitute for the Update function when dealing with Rigibody. The Awake function is called until the script object is loaded. It is used to initialize variábles or statuses before game starts, and is called only once during the lifetime of the script object. The Awake function can use functions such as GameObject.FindWithTag safely because every objects is initialized and called. The Start function is called when the Update function is called first, right before the Update function. It is called only once during the lifetime of the script object, and the Awake function is always called before the Start function.

\subsection{Cases of SNG Unity3D Engine Application}

Still, lots of flashes are being used for SNG development, because a light and smooth engine is appropriate for SNGs considering their characteristics. As the Unity3D engine has come out to the world, the trend of the SNG market is changing. Unity3D can make high-quality games that flashes cannot realize run on the network regardless of types of platforms. Because Unity3D can make high-quality games with low costs due to its characteristic, many game enterprises are expected to use the Unity3D engine in the future, and presently many of them are already using it. 


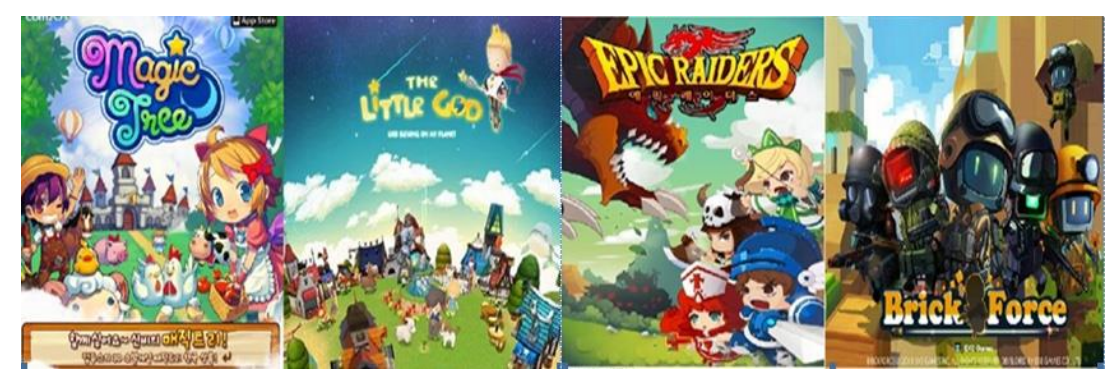

Figure 4. Games Using the Unity3D Engine

\section{Game Design and Development}

\subsection{Structure Map of Game Running}

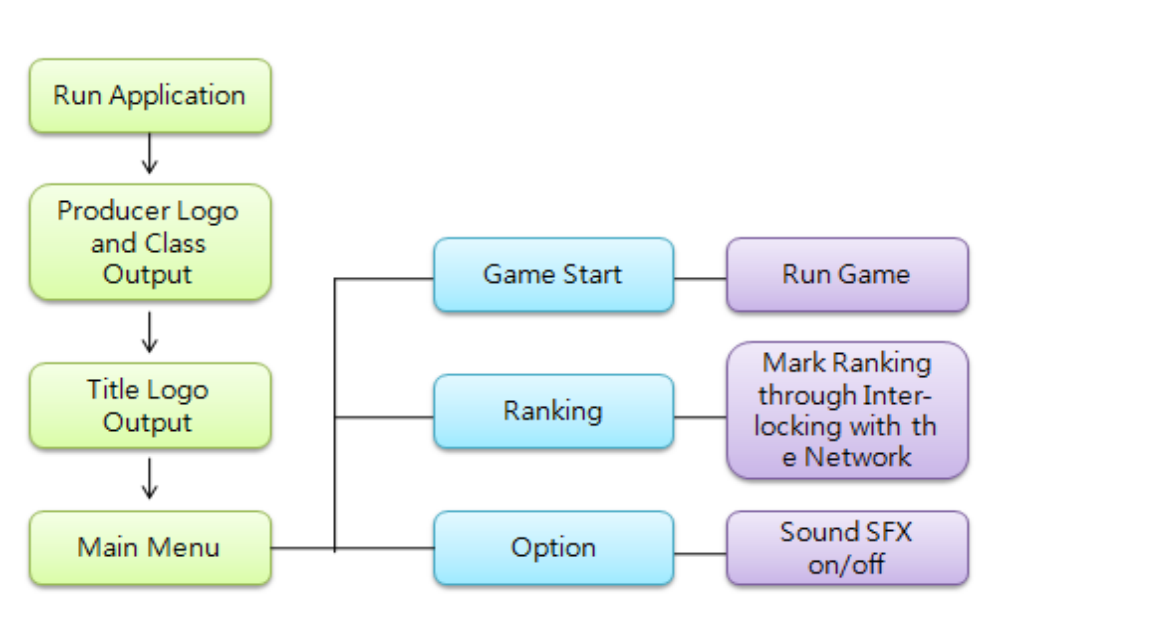

Figure 5. Structure Map of Game Running

(1) Run applieation.-Users run the App by touching the game icon.

(2) Producer logo and gane class output - Output lasts for 3-5 seconds.

(3) Title logo output - Output lasts for 3-5 seconds.

(Add a skip function toy the two outputs when touched, if possible)

(4) Main menu On the first screen that comes out when the processes (1)-(3) are finished are buttons needed for playing the game.

- How to play the game

(1) Run the game and select Game Start on the main menu.

(2) The character stands still at first, and starts to run (climb) when touched.

(3) Keep climbing by doing jumps (one touch) and two-phase jumps (two touches), evading rocks falling from above and thorns sticking out of the wall.

(4) When the character hits an obstacle, game is over and the results window comes out. Total score (height + obtained score) is marked.

(5) This total score is saved in the server, and users can see their scores and their friends' scores on the ranking menu of the main menu.

(6) It is planned to increase the difficulty and fun by updating the character and obstacles afterwards. 


\subsection{Game Graphic Production}

Table. 1 Game Graphic Production

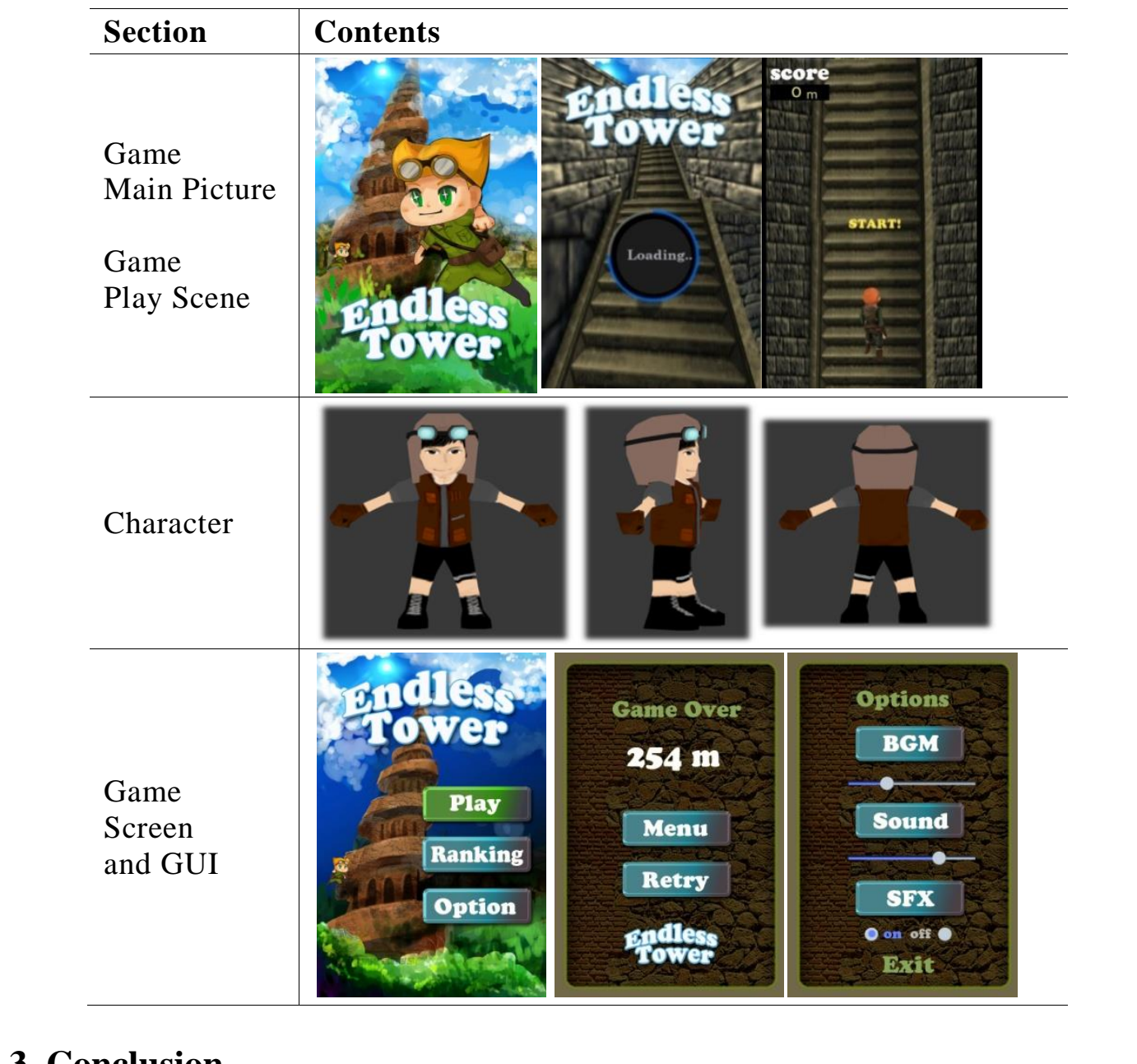

\section{Conclusion}

In the game industuy, core platforms emerged for each time and recently it has changed to a -mylti-platform game and been renovated. Multi-platforms are emerging as an essential strategy for a radical innovation to launch new types of products and maximization of profits.

Especially, as SNS Apps such as KAKAO TLAK or LINE have become prevalent, games linked with theses Apps have achieved huge popularity. Actually, looking at the Android Market, over half of the top 100 games are linked with KAKAO TLAK. A lot of companies currently participating in the market produce many SNG games. It is a big advantage and success factor that the games linked with the social network service are maintained while forming social relations. Although various kinds of engines and tools are used in SNG game production, among them especially the Unity 3D engine through which high-quality games can be created easier and faster, is used in current development by many companies. In particular, it provides many kinds of functions for itself and can produce almost all forms of games. The 3D Unity, a game engine, has a high potential in the efficiency of development process of games. And the increasing availability of it proves its possibility.

The 'Endless Tower' created using the 3D Unity game engine is expected to sufficiently play a role of modern life game which give joy for a while at spare time 
in daily life, through the social functions, easy control and speedy proceeding, and exhibit the potential for developing easier multi-platform games.

\section{References}

[1] Unity Korea, Korean sole distributor and Korean official developers forum "Unity Korea",

[2] http://unity3dkorea.com

[3] R. H. Creighton, "Unity 3D Game Development by Example Beginner's Guide", Acorn, (2011).

[4] H. Youjin, "Status and Prospect of Social Network Game (SNG)", Korean Institute of Industrial Engineer, http://210.101.116.28/kiss2/preview.asp, (2011)

[5] H. Youjin, "Status and Prospect of Social Network Game (SNG)", Korea Creative Content Agency, http://www.kocca.kr/knowledge/trend/internal/1310216_1218.html

[6] Social games, in order to respond to the multi-platform...”, Game Tech, ZDNet Korea,

[7] http://www.zdnet.co.kr/news/news_view.asp?artice_id=20110329160401

[8] R. H. Creighton, "Unity 3D Game Development by Example Beginner's Guide", Acorn (2011)

[9] C. Stevenson and S. Quig, "Unity 3 Blueprints - A Practical Guide to Indie Games Development", Acorn, (2011).

[10] J. Wittayabundit, "Unity 3 Game Development Hotshot", Acorn, (2012).

[11] K. Sangyoon, "3ds max (2013), Digital Books (2012)

[12] J. Bae and A. Kim, "Design and Development of Smart SNGrbased on Unity3D Multi-Platform Game Engine”, Proceedings International Workshop Game and Graphics 2013, (2013).

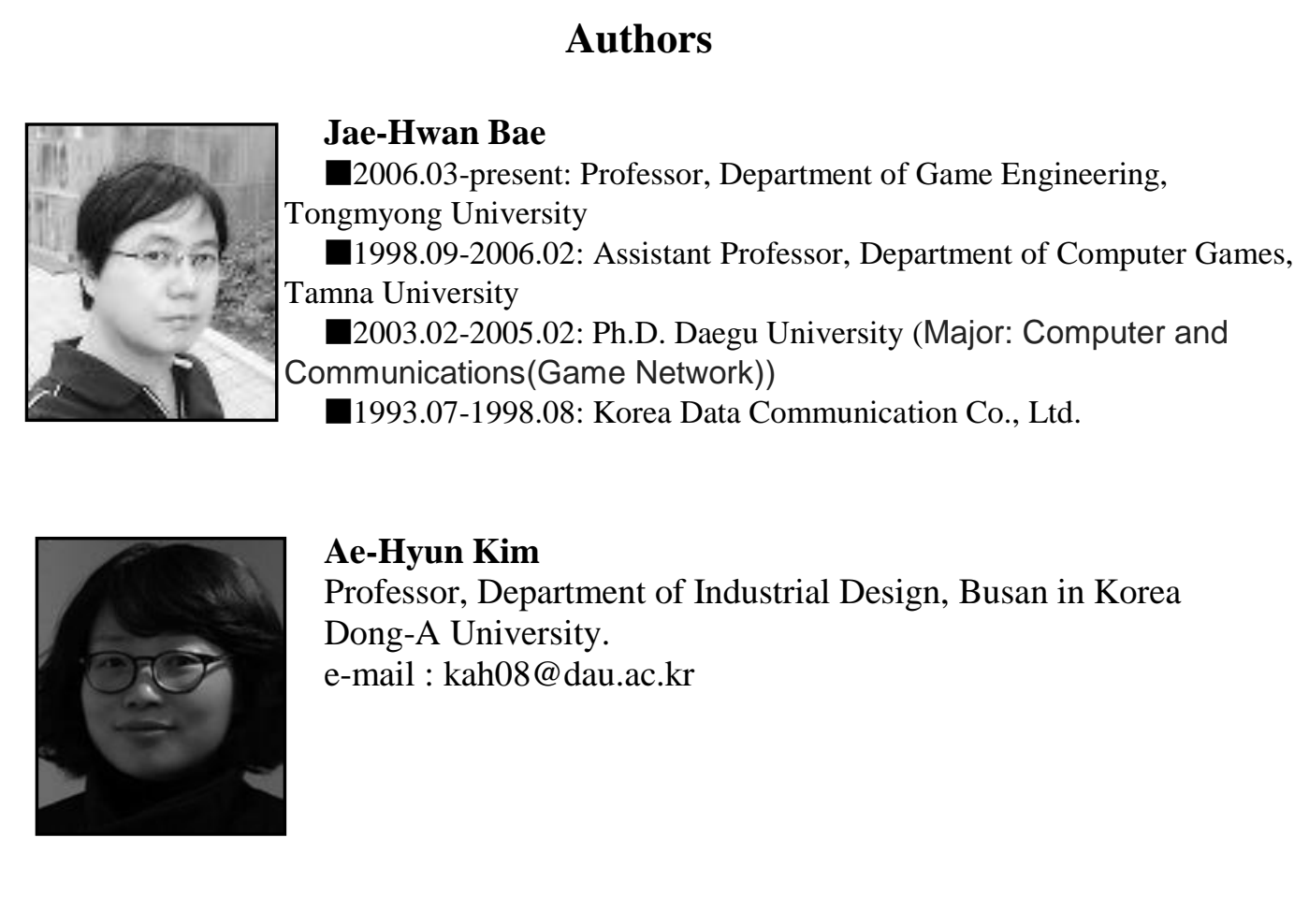

